Manuel Ojanguren · Raman Parimala

\title{
Singularities of generic characteristic polynomials and smooth finite splittings of Azumaya algebras over surfaces
}

Received: 24 October 2008 / Revised: 12 July 2009

Published online: 10 September 2009

\begin{abstract}
Let $k$ be an algebraically closed field. Let $P\left(X_{11}, \ldots, X_{n n}, T\right)$ be the characteristic polynomial of the generic matrix $\left(X_{i j}\right)$ over $k$. We determine its singular locus as well as the singular locus of its Galois splitting. If $X$ is a smooth quasi-projective surface over $k$ and $A$ an Azumaya algebra on $X$ of degree $n$, using a method suggested by M. Artin, we construct finite smooth splittings for $A$ of degree $n$ over $X$ whose Galois closures are smooth.
\end{abstract}

\section{Introduction}

Let $k$ be an algebraically closed field and $A=k\left[X_{i j}, 1 \leq i, j \leq n\right]$ the polynomial ring in $n^{2}$ variables. Let $P(T)=T^{n}+a_{1} T^{n-1}+\cdots+a_{n}$ in $A[T]$ be the characteristic polynomial of the generic matrix $\left(X_{i j}\right)$. We set

$$
A_{n}=A[T] /(P(T)) \text { and } B_{n}=A\left[T_{1}, \ldots, T_{n}\right] / I
$$

where $I$ is the ideal of $A\left[T_{1}, \ldots, T_{n}\right]$ generated by the $n$ polynomials $\sigma_{i}\left(T_{1}, \ldots\right.$, $\left.T_{n}\right)-(-1)^{i} a_{i}, 1 \leq i \leq n$ where for each $i, \sigma_{i}$ is the $i$-th elementary symmetric function. Let $Y_{n}=\operatorname{Spec}\left(A_{n}\right)$ and $Z_{n}=\operatorname{Spec}\left(B_{n}\right)$. In the first part of the paper we describe the singular loci of $Y_{n}$ and $Z_{n}$ and we prove that their codimension is equal to 3. Let $X$ be a smooth quasi-projective surface over $k$. Let $\mathcal{A}$ be an Azumaya algebra of rank $n^{2}$ over $X$. There is a construction due to M. Artin of a degree $n$ finite flat map $Y \rightarrow X$ with $Y$ smooth which splits $\mathcal{A}$ (cf [8] for the case $X$ projective and $\mathcal{A}$ generically a division ring). We use the method of proof in [8] to construct a degree $n$ flat map $Y \rightarrow X$ which splits $\mathcal{A}$ where $Y$ is smooth and has a smooth irreducible Galois closure.

\section{The characteristic polynomial of the generic matrix}

In this section we suppose that $k$ is an algebraically closed field, of arbitrary characteristic. We denote by $\operatorname{Sing}(\mathrm{X})$ the singular locus of a given scheme $X$.

M. Ojanguren ( $\varangle$ ): IGAT, EPFL, 1015, Lausanne, Switzerland e-mail: manuel.ojanguren@epfl.ch

R. Parimala: Department of Mathematics and Computer Science, Emory University, 400 Dowman Drive, Atlanta, GA, USA

e-mail: parimala@mathcs.emory.edu

Mathematics Subject Classification (2000): Primary 16H05; Secondary 14F22 
Let

$$
A_{n}=\frac{k\left[X_{11}, X_{12}, \ldots, X_{n n}\right][T]}{(P(T))}
$$

where $P(T)$ is the characteristic polynomial of the generic matrix $\left(X_{i j}\right)$ with $1 \leq$ $i, j \leq n$. Let $Y_{n}=\operatorname{Spec}\left(A_{n}\right)$. We study the singular locus of $Y_{n}$.

Lemma 1.1. Let $\beta=\operatorname{diag}\left(\mathrm{B}_{1}, \ldots, \mathrm{B}_{\mathrm{m}}\right)$ be a matrix consisting of $m$ cyclic Jordan blocks

$$
B_{i}=\left(\begin{array}{cccccccc}
\lambda_{i} & 1 & 0 & . & . & . & 0 & 0 \\
0 & \lambda_{i} & 1 & . & . & . & 0 & 0 \\
0 & 0 & \lambda_{i} & . & . & . & 0 & 0 \\
. & . & . & . & . & . & . & . \\
0 & 0 & 0 & . & . & . & \lambda_{i} & 1 \\
0 & 0 & 0 & . & . & . & 0 & \lambda_{i}
\end{array}\right)
$$

with distinct eigenvalues $\lambda_{i}$. Then, for any $i$, the scheme $Y_{n}$ is smooth at $\left(\beta, \lambda_{i}\right)$.

Proof. We denote by $\mathrm{I}_{n}$ the identity matrix of size $n$. Developing the determinant of $\left(X_{i j}\right)-T \cdot \mathrm{I}_{n}$ along the first column we get

$$
\pm P(T)=\left(X_{11}-T\right) P_{1}(T)+X_{2,1} P_{2}(T)+\cdots+X_{n, 1} P_{n}(T)
$$

where the polynomials $P_{i}$ are the cofactors of the first column. Let $k_{i}$ be the size of $B_{i}$. We see that $P_{k_{1}}(T)\left(B, \lambda_{1}\right)$ is (up to sign) the determinant of a matrix of the form $\operatorname{diag}\left(\mathrm{I}_{\mathrm{k}_{1}-1}, \mathrm{~B}_{2}-\lambda_{1} \mathrm{I}_{\mathrm{k}_{2}}, \ldots, \mathrm{B}_{\mathrm{m}}-\lambda_{1} \mathrm{I}_{\mathrm{k}_{\mathrm{m}}}\right)$, it being understood that the first block is missing if $k_{1}=1$. Since $\lambda_{1} \neq \lambda_{i}$, this shows that $\partial P(T) / \partial X_{k_{1}, 1}=P_{k_{1}}(T)$ is not zero at $\left(B, \lambda_{1}\right)$. Thus $Y_{n}$ is smooth at $\left(\beta, \lambda_{1}\right)$ and the same clearly holds for any other $\lambda_{i}$.

Lemma 1.2. Every neighbourhood of a matrix $\alpha$ with an eigenvalue $\lambda \neq 0$ contains an invertible semisimple matrix with eigenvalue $\lambda$.

Proof. We may assume that $\alpha$ is in Jordan form. The given neighbourhood of $\alpha$ contains an open set defined by the non-vanishing of a polynomial $g$ in the coordinates of the generic matrix $\left(X_{i j}\right)$. We may assume that the diagonal entries of $\alpha$ are $\left(\lambda, \lambda_{2}, \ldots, \lambda_{n}\right)$. Since $g(\alpha) \neq 0$ we may find values $\lambda_{2}^{\prime}, \ldots, \lambda_{n}^{\prime}$ all distinct and different from $\lambda$ and different from 0 , such that when we replace $\lambda_{i}$ by $\lambda_{i}^{\prime}$ in $\alpha$ we obtain an $\alpha^{\prime}$ for which $g\left(\alpha^{\prime}\right) \neq 0$. This new $\alpha^{\prime}$ is in the given neighbourhood and is semisimple.

Let $Y_{n}$ be as before. The surjection $k\left[X_{11}, X_{12}, \ldots, X_{n n}\right][T] \rightarrow A_{n}$ induces a finite map $\pi: Y_{n} \rightarrow \mathbb{A}_{k}^{n^{2}}$. The projection $C=\pi\left(\operatorname{Sing}\left(Y_{n}\right)\right)$ is a closed subscheme of $\mathbb{A}_{k}^{n^{2}}$ and is contained in the ramification locus of $\pi$, which is the closed subscheme of $\mathbb{A}_{k}^{n^{2}}$ whose closed points correspond to matrices with at least two equal eigenvalues. 
Lemma 1.3. Let $V \subset \mathbb{A}_{k}^{n^{2}}$ be the set of semisimple invertible matrices with at least two coincident eigenvalues. Then $V \subseteq C$.

Proof. It suffices to check that any matrix of the form $\beta=\operatorname{diag}\left(\mu_{1}, \ldots, \mu_{\mathrm{n}-2}, \lambda, \lambda\right)$ is in $C$. We show that $(\beta, \lambda)$ belongs to $\operatorname{Sing}\left(Y_{n}\right)$. Writing $X_{i i}=\mu_{i}+X_{i}$ for $i \leq n-2, X_{i i}=\lambda+X_{i}$ for $i \geq n-1, T=\lambda+t$ and $v_{i}=\mu_{i}-\lambda$ we see that $\pm P(T)$ is the determinant of the matrix

$$
\left(\begin{array}{cccc}
v_{1}+X_{1}-t & X_{12} & \cdots & X_{1 n} \\
X_{2,1} & v_{2}+X_{2}-t & \cdots & X_{2, n} \\
\cdots & \cdots & \cdots & \cdots \\
\cdots & \cdots & X_{n-1}-t & X_{n-1, n} \\
\cdots & \cdots & X_{n, n-1} & X_{n}-t
\end{array}\right)
$$

and it is clear that it does not contain any linear term in $X_{i}, X_{i j}$ or $T$. Thus the variety it defines is singular at the origin, which corresponds to the point $(\beta, \lambda)$ in the previous coordinates.

Let $P_{n}$ be the affine space of monic polynomials of degree $n$. Let $c: M_{n} \rightarrow P_{n}$ be the characteristic polynomial map associating to any $n \times n$-matrix its characteristic polynomial. We have the finite surjective map $\sigma: \mathbb{A}_{k}^{n} \rightarrow P_{n}$ sending $\xi=\left(\xi_{1}, \ldots, \xi_{n}\right)$ to the polynomial $T^{n}+\sigma_{1}(\xi) T^{n-1}+\cdots+\sigma_{n}(\xi)$, where, for $1 \leq i \leq n, \sigma_{i}$ is the $i$-th elementary symmetric function. For a given positive integer $l \leq n$, the set of polynomials in $P_{n}$ with at least $l$ distinct eigenvalues is an open dense subscheme of $P_{n}$.

Lemma 1.4. Let $W \subset M_{n}(k)$ be the set of all semisimple invertible matrices with at least $n-1$ distinct eigenvalues. Then $W$ is open and dense in $M_{n}(k)$.

Proof. The set $M$ of all semisimple invertible matrices is open and dense in $M_{n}(k)$. The set $P$ of all the polynomials in $P_{n}(k)$ which have at least $n-1$ distinct eigenvalues is open and dense. Hence $W=M \cap c^{-1}(P)$ is open and dense in $M_{n}(k)$.

By 1.4 the set $U=W \cap C$ of all semisimple invertible matrices with exactly $n-1$ distinct eigenvalues is open in $C$.

Lemma 1.5. The set $U$ is dense in $C$.

Proof. Let $(\beta, \lambda)$ be a point of $\operatorname{Sing}\left(Y_{n}\right)$. By $1.1, \beta$, which we may assume to be in Jordan canonical form, contains at least two cyclic Jordan blocks with the same eigenvalue. We write $\beta=\operatorname{diag}\left(\beta_{1}, \beta_{2}, \ldots, \beta_{\mathrm{r}}\right)$ with the $\beta_{i}$ 's cyclic Jordan blocks of size $s_{i}$ and $\beta_{1}, \beta_{2}$ having the same eigenvalue $\lambda$. Suppose that $\beta$ is in the open set defined by $f \neq 0$ for some polynomial function $f$ in the entries $X_{i j}$ of the generic $n \times n$ matrix. Let $\widetilde{\beta}=\operatorname{diag}\left(\widetilde{\beta}_{1}, \widetilde{\beta}_{2}, \ldots, \widetilde{\beta}_{\mathrm{r}}\right)$ be a matrix where each $\widetilde{\beta}_{i}$ has the same size as $\beta_{i}$ and the same off-diagonal entries. Suppose further that $\widetilde{\beta}$ has $n-1$ distinct eigenvalues, with $\widetilde{\beta}_{1}$ and $\widetilde{\beta}_{2}$ retaining the eigenvalue $\lambda$. Then $\widetilde{\beta}$ is semisimple and, for a general $\widetilde{\beta}, f(\widetilde{\beta}) \neq 0$. 
For example, if

$$
\beta=\left(\begin{array}{lllll}
\lambda & 1 & 0 & 0 & 0 \\
0 & \lambda & 1 & 0 & 0 \\
0 & 0 & \lambda & 0 & 0 \\
0 & 0 & 0 & \lambda & 1 \\
0 & 0 & 0 & 0 & \lambda
\end{array}\right)
$$

then

$$
\widetilde{\beta}=\left(\begin{array}{lllll}
\lambda_{1} & 1 & 0 & 0 & 0 \\
0 & \lambda_{2} & 1 & 0 & 0 \\
0 & 0 & \lambda & 0 & 0 \\
0 & 0 & 0 & \lambda_{3} & 1 \\
0 & 0 & 0 & 0 & \lambda
\end{array}\right)
$$

with $\lambda, \lambda_{1}, \lambda_{2}, \lambda_{3}$ distinct.

Corollary 1.6. The dimension of $C$ is equal to the dimension of $U$.

Lemma 1.7. The dimension of $U$ is $n^{2}-3$.

Proof. Let $\Sigma_{n-1} \subset P_{n}$ be the subset of polynomials having $n-1$ distinct roots. Then $\Sigma_{n-1}$, being the image under $\sigma$ of a closed subset of dimension $n-1$, has dimension $n-1$. The restriction of $c$ to $U$ yields a surjective map $c_{U}: U \rightarrow \Sigma_{n-1}$. The linear group $G L_{n}(k)$ acts by conjugation transitively on each fibre of $c_{U}$ and the stabilizer of the matrix $\operatorname{diag}\left(\lambda, \lambda, \lambda_{3}, \ldots, \lambda_{\mathrm{n}}\right)$ is $G L_{2}(k) \times\left(k^{*}\right)^{n-2}$. Hence the dimension of $U$ is $\operatorname{dim}\left(G L_{n}(k)\right)-\operatorname{dim}\left(G L_{2}(k) \times\left(k^{*}\right)^{n-2}\right)+\operatorname{dim}\left(\Sigma_{n-1}\right)=$ $n^{2}-(4+n-2)+n-1=n^{2}-3$.

Corollary 1.8. The closed set $\operatorname{Sing}\left(Y_{n}\right)$ is of codimension 3.

Proof. The closure of $U$ is $C=\pi\left(\operatorname{Sing}\left(Y_{n}\right)\right)$ and $\pi$ is a finite map.

\section{The generic Galois closure}

Let $X_{i j}$ with $i, j$ running from 1 to $n$ be indeterminates and write $P(T)=T^{n}+$ $a_{1} T^{n-1}+\cdots+a_{n}$ for the characteristic polynomial of the generic matrix $\left(X_{i j}\right)$. Let $A$ be the polynomial $k$-algebra in the $X_{i j}$. Consider another set $T_{1}, \ldots, T_{n}$ of indeterminates and let

$$
B_{n}=A\left[T_{1}, \ldots, T_{n}\right] / I
$$

where $I$ is the ideal generated by all the polynomials $\sigma_{i}\left(T_{1}, \ldots, T_{n}\right)-(-1)^{i} a_{i}$ for $1 \leq i \leq n$. Let $Z_{n}=\operatorname{Spec}\left(B_{n}\right)$. We want to determine $\operatorname{Sing}\left(Z_{n}\right)$.

A $k$-point of $Z_{n}$ is a pair $(\alpha, t)$ with the characteristic polynomial of $\alpha$,

$$
P(\alpha)(T)=T^{n}+a_{1}(\alpha) T^{n-1}+\cdots+a_{n}(\alpha)
$$


satisfying $a_{i}(\alpha)=\sigma_{i}(t), 1 \leq i \leq n$.

Let $\pi: Z_{n} \rightarrow \operatorname{Spec}(A)$ be the first projection and let $S=\pi\left(\operatorname{Sing}\left(Z_{n}\right)\right)$. We want to compute the dimension of $S$.

Let $(\alpha, t)$ be a singularity of $Z_{n}$. Since no $\sigma_{i}\left(T_{1}, \ldots, T_{n}\right)$ involves the $X_{i j}$ and no $a_{j}$ involves the $T_{i}$, if we order the $X_{i j}$ lexicographically, the Jacobian matrix of the equations $\sigma_{i}\left(T_{1}, \ldots, T_{n}\right)-(-1)^{i} a_{i}=0$ is of size $\left(n^{2}+n\right) \times n$ and looks as follows:

$$
J=\left(\begin{array}{ccc}
\frac{\partial \sigma_{1}}{\partial T_{1}} & \cdots & \frac{\partial \sigma_{n}}{\partial T_{1}} \\
\vdots & & \vdots \\
\frac{\partial \sigma_{1}}{\partial T_{n}} & \cdots & \frac{\partial \sigma_{n}}{\partial T_{n}} \\
\frac{\partial a_{1}}{\partial X_{11}} & \cdots & \frac{(-1)^{n-1} \partial a_{n}}{\partial X_{11}} \\
\vdots & & \vdots \\
\frac{\partial a_{1}}{\partial X_{n n}} & \cdots & \frac{(-1)^{n-1} \partial a_{n}}{\partial X_{n n}}
\end{array}\right) .
$$

Since $\pi$ is a finite map, the dimension of $Z_{n}$ is $n^{2}$. The point $(\alpha, t)$ being a singularity of $Z_{n}$, the Jacobian criterion implies that the rank of $J$ at $(\alpha, t)$ is at most $n-1$. Thus, in particular, the determinant $\delta$ of the top $n \times n$ block of $J$ must vanish at $(\alpha, t)$. It is well-known that $\delta= \pm \prod_{i<j}\left(T_{i}-T_{j}\right)$. This shows that $\alpha$ has at least two equal eigenvalues. In other words, denoting by $V(-)$ the vanishing locus of a given set of polynomials, $(\alpha, t)$ belongs to the vanishing locus $V\left(\delta^{2}\right)$ of the discriminant $\delta^{2}$ of $P(T)$.

Consider now $\operatorname{Sing}\left(Z_{n}\right) \cap V\left(a_{1}, \ldots, a_{n}\right)$. Since $\operatorname{Sing}\left(Z_{n}\right) \subset V\left(\delta^{2}\right)$ we have

$$
\operatorname{Sing}\left(Z_{n} \cap V\left(a_{1}, \ldots, a_{n}\right)\right)=\operatorname{Sing}\left(Z_{n} \cap V\left(\delta^{2}, a_{1}, \ldots, a_{n}\right)\right) .
$$

But the vanishing of $a_{1}, \ldots, a_{n-1}$ and $\delta^{2}$ already implies the vanishing of $a_{n}$; in fact, if $T^{n}-a_{n}$ has a multiple root, then $a_{n}=0$ (we are in characteristic 0 ). Thus

$$
\operatorname{Sing}\left(Z_{n}\right) \cap V\left(a_{1}, \ldots, a_{n-1}\right)=\operatorname{Sing}\left(Z_{n}\right) \cap V\left(a_{1}, \ldots, a_{n}\right)
$$

and therefore

$$
\operatorname{dim}\left(\operatorname{Sing}\left(Z_{n}\right)\right) \leq \operatorname{dim}\left(\operatorname{Sing}\left(Z_{n}\right) \cap V\left(a_{1}, \ldots, a_{n}\right)\right)+n-1 .
$$

The set $V\left(a_{1}, \ldots, a_{n}\right)$ is the set $\mathcal{N}$ of nilpotent matrices. On the other hand, the bottom block of the Jacobian matrix must have rank at most $n-1$, which means that $\alpha$ is a singular point of $\mathcal{N}$. This shows that $\operatorname{Sing}\left(Z_{n}\right) \cap \mathcal{N} \subseteq \operatorname{Sing}(\mathcal{N})$ and from the previous inequality we obtain the next result.

Lemma 2.4. The dimension of $\operatorname{Sing}\left(Z_{n}\right)$ is at most $\operatorname{dim}(\operatorname{Sing}(\mathcal{N}))+n-1$.

We now compute the dimension of $\operatorname{Sing}(\mathcal{N})$. As pointed out by George McNinch, our computation could be deduced from results already in the literature (see for instance [7], Sect.7) but we prefer to be as self-contained as possible. We begin with the computation of the dimension of $\mathcal{N}$. 
Proposition 2.5. Let $\mathcal{N} \subset M_{n}$ denote the variety of nilpotent matrices. Then the dimension of $\mathcal{N}$ is $n^{2}-n$.

Proof. Since $\mathcal{N}$ is defined by the ideal $\left(a_{1}, \ldots, a_{n}\right)$ of $A=k\left[X_{11}, X_{12}, \ldots, X_{n n}\right]$, it suffices to show that this ideal has height $n$. Let $I$ be the ideal generated by

$$
\left(a_{1}, \ldots a_{n}, X_{i j} \mid i \neq j\right) \text {. }
$$

We claim that this ideal has height $n^{2}$. The ring $A / I$ is isomorphic to

$$
k\left[X_{11}, X_{2,2}, \ldots, X_{n n}\right] / J
$$

where $J$ is the ideal generated by the elementary symmetric functions $\sigma_{1}, \ldots, \sigma_{n}$ in $X_{11}, X_{2,2}, \ldots, X_{n n}$. Since $k\left[X_{11}, \ldots, X_{n n}\right]$ is finite over $k\left[\sigma_{1}, \ldots, \sigma_{n}\right]$, the ideal $J$ has height $\mathrm{n}$ in $k\left[X_{11}, \ldots, X_{n n}\right]$. Hence $I$ is supported only at closed points. Since the $a_{i}$ are homogeneous, it follows that the ideal $\left(a_{1}, \ldots, a_{n}\right)$ has height $\mathrm{n}$.

Lemma 2.6. A nilpotent matrix $\alpha$ whose Jordan form consists of only one cyclic block is not a singularity of $\mathcal{N}$. More precisely, the determinant of $\left(\frac{\partial a_{i}}{\partial X_{j 1}}\right)$ is not zero at $\alpha$.

Proof. Let $A$ be as before and $P(T)=T^{n}+a_{1} T^{n-1}+\cdots+a_{n}$ the characteristic polynomial of the generic matrix $\left(X_{i j}\right)$. The variety of nilpotent matrices is $\mathcal{N}=V\left(a_{1}, \ldots, a_{n}\right)$. We show that at

$$
\alpha=\left(\begin{array}{cccccccc}
0 & 1 & 0 & . & . & . & 0 & 0 \\
0 & 0 & 1 & . & . & . & 0 & 0 \\
0 & 0 & 0 & . & . & . & 0 & 0 \\
. & . & . & . & . & . & . & . \\
0 & 0 & 0 & . & . & . & 0 & 1 \\
0 & 0 & 0 & . & . & . & 0 & 0
\end{array}\right)
$$

the jacobian matrix $\left(\frac{\partial a_{i}}{\partial X_{j k}}\right)$ has rank $n$. We compute the $n \times n$ matrix $\left(\frac{\partial a_{i}}{\partial X_{j 1}}\right)$. The derivative of $a_{i}$ by $X_{j 1}$ is the coefficient of $T^{n-i}$ in $\frac{\partial P(T)}{\partial X_{j 1}}$. Developing the determinant of $\left(X_{i j}\right)-T \mathrm{I}_{n}$ along the first column we find

$$
\pm P(T)=\left(X_{11}-T\right) P_{1}(T)+X_{2,1} P_{2}(T)+\cdots+X_{n, 1} P_{n}(T)
$$

where $P_{i}(T)$ is the determinant of an $(n-1) \times(n-1)$ matrix $M_{i}$. At $\left(X_{i j}\right)=\alpha$ we find

$$
M_{i}(\alpha)=\left(\begin{array}{ll}
B_{1} & 0 \\
0 & B_{2}
\end{array}\right)
$$

with

$$
B_{1}=\left(\begin{array}{cccccccc}
1 & 0 & 0 & \cdot & . & . & 0 & 0 \\
-T & 1 & 0 & . & . & . & 0 & 0 \\
0 & -T & 1 & . & . & . & 0 & 0 \\
. & . & . & . & . & . & . & . \\
0 & 0 & 0 & . & . & . & 1 & 0 \\
0 & 0 & 0 & . & . & . & -T & 1
\end{array}\right)
$$


of size $j-1$ and

$$
B_{2}=\left(\begin{array}{cccccccc}
-T & 1 & 0 & . & . & . & 0 & 0 \\
0 & -T & 1 & . & . & . & 0 & 0 \\
0 & 0 & -T & . & . & . & 0 & 0 \\
. & . & . & . & . & . & . & . \\
0 & 0 & 0 & . & . & \cdot & -T & 1 \\
0 & 0 & 0 & . & . & . & 0 & -T
\end{array}\right)
$$

of size $n-j$. Thus $P_{j}(T)= \pm T^{n-j}$ and $\frac{\partial a_{i}}{\partial X_{j 1}}(\alpha)$ is \pm 1 for $j=i$ and zero otherwise. This proves the lemma.

Lemma 2.7. The set $\mathcal{N}_{2}$ of nilpotent matrices whose Jordan form has exactly two cyclic blocks are dense in the set of nilpotent matrices whose Jordan form has two or more blocks.

Proof. Let $\alpha=\operatorname{diag}\left(B_{1}, B_{2}, \ldots, B_{m}\right)$ be a nilpotent matrix which we can assume to be in Jordan form with blocks $B_{1}, \ldots, B_{m}, m \geq 3$. Let $g \neq 0$ with $g \in A$ define a neighbourhood of $\alpha$. We can find constants $\epsilon_{2}, \ldots, \epsilon_{m-1}$ such that replacing the zeros between the superdiagonals of $B_{2}$ and $B_{3}$, between the superdiagonals $B_{3}$ and $B_{4}$ and so on, by the $\epsilon_{i}$ we obtain a matrix $\alpha^{\prime}$ such that $g\left(\alpha^{\prime}\right) \neq 0$. Clearly $\alpha^{\prime}$ has two cyclic blocks.

Lemma 2.8. If $\alpha \in \mathcal{N}$ has a Jordan form with two or more cyclic blocks, then $\alpha$ is a singularity of $\mathcal{N}$.

Proof. We may assume that $\alpha$ is in Jordan form and can be written as

$$
\operatorname{diag}\left(B_{1}, B_{2}, \ldots, B_{m}\right)
$$

where $m \geq 2$, each $B_{i}$ is a cyclic Jordan block, $B_{1}$ is of size $p$ and $B_{2}$ of size $q$. We can write the generic matrix as $\left(X_{i j}\right)=\left(\alpha+Y_{i j}\right)$. Then $\frac{\partial a_{i}}{\partial X_{i j}}(\alpha)=\frac{\partial a_{i}}{\partial Y_{i j}}(0)$. But in the matrix $\alpha+\left(Y_{i j}\right)$ the $p$-th line and the $(p+q)$-th line are linear homogeneous in the $Y_{i j}$, hence developing the determinant of $\alpha+\left(Y_{i j}\right)$ along these two lines we see that $a_{n}\left(Y_{i j} \mid 1 \leq i, j \leq n\right)$ has no constant and no linear term. This shows that all the derivatives $\frac{\partial a_{n}}{\partial Y_{i j}}$ vanish at the origin and therefore the Jacobian matrix $\frac{\partial a_{i}}{\partial Y_{i j}}$ cannot be of rank $n$.

Corollary 2.9. The set $\mathcal{N}_{2}$ is dense in $\operatorname{Sing}(\mathcal{N})$.

The set $\mathcal{N}_{2}$ is the union of the $G L_{n}(k)$-orbits $S_{p, q}$ of all the matrices of the form $\beta=\operatorname{diag}\left(B_{p}, B_{q}\right)$ where $B_{p}$ is the nilpotent cyclic Jordan block of size $p$ and $B_{q}$ the nilpotent cyclic Jordan block of size $q=n-p$. In particular, it is the finite union of the constructible sets $S_{p, q}$. The dimension of $S_{p, q}$ is $n^{2}-s$ where $s$ is the dimension of the isotropy group of $\beta$.

Lemma 2.10. The dimension of the isotropy group of $\operatorname{diag}\left(B_{p}, B_{q}\right)$ is

$$
p+q+2 \min (p, q) .
$$

In particular it is always at least $p+q+2$. 
Proof. Let $\Gamma \subset G L_{n}(K)$ be the isotropy group of $\beta=\operatorname{diag}\left(B_{p}, B_{q}\right)$. Let

$$
\gamma=\left(\begin{array}{ll}
A & B \\
C & D
\end{array}\right)
$$

be an element of $\Gamma$, written with blocks $A, B, C, D$ of suitable sizes. The condition $\gamma \beta \gamma^{-1}=\beta$ is equivalent to the conditions

$$
A B_{p}=B_{p} A, D B_{q}=B_{q} D, B B_{q}=B_{p} B, C B_{p}=B_{q} C .
$$

We compute the dimension of the linear subspace $\Gamma_{0}$ of $M_{p+q}(K)$ consisting of matrices that satisfy the four conditions above.

An explicit matrix computation shows that the first condition gives

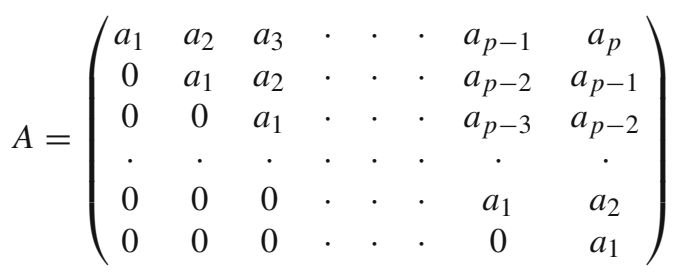

A similar result holds for $D$, hence the matrices $\operatorname{diag}(A, D)$ in $\Gamma_{0}$ span a linear space of dimension $p+q$.

Assume now that $p \leq q$. An explicit computation shows that the third condition gives

$$
B=\left(\begin{array}{ccccccccccccc}
0 & . & . & . & 0 & b_{1} & b_{2} & b_{3} & . & . & . & b_{p-1} & b_{p} \\
0 & . & . & . & 0 & 0 & b_{1} & b_{2} & . & . & . & b_{p-2} & b_{p-1} \\
0 & . & . & . & 0 & 0 & 0 & b_{1} & . & . & . & b_{p-3} & b_{p-2} \\
. & . & . & . & . & . & . & . & . & . & . & . & . \\
. & . & . & . & . & . & . & . & . & . & . & . & . \\
0 & . & . & . & . & . & . & . & . & . & . & b_{1} & b_{2} \\
0 & . & . & . & . & . & . & . & . & . & . & 0 & b_{1}
\end{array}\right)
$$

A similar result holds for $C$, hence, when $p \leq q$ the dimension of $\Gamma_{0}$ is $p+q+$ $p+p=p+q+2 \min (p, q)$ and clearly this is also the dimension (as a variety) of $\Gamma$.

Proposition 2.11. For $n \geq 3$ the dimension of $\operatorname{Sing}(\mathcal{N})$ is $n^{2}-n-2$.

Proof. By 2.9 and 2.10, $\operatorname{dim}(\operatorname{Sing}(\mathcal{N}))=\operatorname{dim}\left(\mathcal{N}_{2}\right)=n^{2}-\min _{p, q}\left(\operatorname{dim}\left(S_{p, q}\right)\right)$. The isotropy group of minimal dimension is $S_{1, n-1}$ which has dimension $n+2$. Thus $\operatorname{dim}\left(\mathcal{N}_{2}\right)=n^{2}-(n+2)$.

Theorem 2.12. For $n \geq 3$ the dimension of $\operatorname{Sing}\left(Z_{n}\right)$ is at most $n^{2}-3$.

Proof. This immediately follows from 2.4 and 2.11 . 


\section{Finite splitting of Azumaya algebras}

Let $X$ be a smooth quasi-projective irreducible surface over an algebraically closed field $k, K=k(X)$ the field of rational functions of $X$ and $A$ a central simple algebra of degree $n$ over $K$. Let $\mathcal{A}$ be a maximal order in $A$ defined over $X$. We do not assume that $A$ is a division ring.

Lemma 3.1. There exists an element $\sigma$ in A whose characteristic polynomial is irreducible, separable and has Galois group $\mathcal{S}_{n}$.

Proof. Let $\sigma_{1}, \ldots, \sigma_{m}$ be a $K$-basis of $A$ ( $m$ being equal to $n^{2}$ ). Let $K \subset L$ be a separable finite extension of $K$ such that $A \otimes_{K} L=M_{n}(L)$. Let $X_{1}, \ldots, X_{m}$ be indeterminates and $\tilde{\sigma}=X_{1} \sigma_{1}+\cdots+X_{m} \sigma_{m}$. After an $L$-linear change of variables the characteristic polynomial $P_{\widetilde{\sigma}}(T)$ of $\widetilde{\sigma}$ is the characteristic polynomial of the generic matrix, hence it is irreducible and separable over $L\left(X_{1}, \ldots, X_{m}\right)$, and has Galois group $\mathcal{S}_{n}$. Since it is defined over $K\left(X_{1}, \ldots, X_{m}\right)$ it has the same properties over this smaller field. By Hilbert's irreducibility theorem (see for instance [4], Proposition 16.1.5) there exist $\xi_{1}, \ldots, \xi_{m}$ in $K$ such that the characteristic polynomial of $\sigma=\xi_{1} \sigma_{1}+\cdots+\xi_{m} \sigma_{m}$ is irreducible, separable, with Galois group $\mathcal{S}_{n}$.

We fix a smooth embedding of $X$ in a projective space. If $d$ is sufficiently large, the twisted sheaf $\mathcal{A}(d)$ is generated by global sections $s_{1}, \ldots s_{N}$. For $\sigma$ as in Lemma 1 and a suitable global section $g$ of $\mathcal{O}_{X}(d), \sigma g$ is a global section of $\mathcal{A}(d)$ and we may assume that $s_{N}=\sigma g$. Such a set of global sections will be called admissible. We set $\mathcal{L}=\mathcal{O}_{X}(d)$.

Let $s$ be any global section of $\mathcal{A}(d)=\mathcal{A} \otimes_{\mathcal{O}_{X}} \mathcal{L}$. Choose an arbitrary affine nonempty open set $U \subset X$ over which $\mathcal{L}$ is principal: $\mathcal{L}_{\mid U}=\mathcal{O}_{U} f$ for some $f \in \mathcal{L}(U)$. Then $s f^{-1} \in \mathcal{A}(U)$, which is a maximal order over $\mathcal{O}_{X}(U)$. Let

$$
P_{f, U}(T)=T^{n}+b_{1} T^{n-1}+\cdots+b_{n}
$$

with $b_{1}, \ldots, b_{n} \in k[U]$ be the characteristic polynomial of $s f^{-1}$. We define $J_{f, U}$ as the ideal of

$\operatorname{Sym}\left(\left.\mathcal{L}^{-1}\right|_{U}\right)=\left.\left.\mathcal{O}_{U} \oplus \mathcal{L}^{-1}\right|_{U} \oplus \mathcal{L}^{-2}\right|_{U} \oplus \cdots=\mathcal{O}_{U} \oplus \mathcal{O}_{U} f^{-1} \oplus \mathcal{O}_{U} f^{-2} \oplus \cdots$

generated by $f^{-n} \oplus b_{1} f^{-(n-1)} \oplus \cdots \oplus b_{n}$.

Lemma 3.2. Let $\Lambda$ be a central simple algebra of rank $n^{2}$ over a field $K$. For any $\alpha \in \Lambda$ and any $c \in K$, the characteristic polynomial $P_{\alpha}(T)$ of $\alpha$ satisfies the relation $c^{n} P_{\alpha}(T)=P_{c \alpha}(c T)$.

Proof. It immediately follows from the split case $\Lambda=M_{n}(K)$.

Lemma 3.3. The ideal $J_{f, U}$ does not depend on the choice of $f$. 
Proof. We apply 3.2 with $f=u g$ for some other generator $g$ of $\left.\mathcal{L}\right|_{U}$ and $u$ invertible on $U$. (We note that the suffixes $f$ or $g$ stand for the elements $s / f, s / g$ in the algebra). We have

$$
P_{g, U}(T)=P_{u^{-1} f, U}(T)=u^{n} P_{f, U}\left(u^{-1} T\right)=T^{n}+u b_{1} T^{n-1}+\cdots+u^{n} b_{n} .
$$

Thus the ideal $J_{g, U}$ is generated by

$$
g^{-n} \oplus b_{1} u g^{-(n-1)} \oplus \cdots \oplus u^{n} b_{n}=u^{n}\left(f^{-n} \oplus b_{1} f^{-(n-1)} \oplus \cdots \oplus b_{n}\right) .
$$

and coincides therefore with $J_{f, U}$.

Patching the ideals $J_{f, U}$ over a suitable affine covering of $X$ yields a global ideal $J_{s}$ of $\operatorname{Sym}\left(\mathcal{L}^{-1}\right)$ that only depends on the section $s$. We call $J_{s}$ the characteristic ideal of $s$.

The ideal $J_{S}$ defines a closed subscheme $Y_{S}$ of $\operatorname{Spec}\left(\operatorname{Sym}\left(\mathcal{L}^{-1}\right)\right)$ which is clearly finite and flat over $X$.

To simplify notation, if $s=\lambda_{1} s_{1}+\cdots+\lambda_{N} s_{N}$ we put $\lambda=\left(\lambda_{1}, \ldots, \lambda_{N}\right) \in k^{N}$, $J_{s}=J_{\lambda}$ and $Y_{s}=Y_{\lambda}$. We denote by $\pi_{\lambda}: Y_{\lambda} \rightarrow X$ the natural map.

Theorem 3.4. Let X be a smooth quasi-projective irreducible surface over an algebraically closed field $k, K=k(X)$ the field of rational functions of $X$ and $A$ a central simple algebra of degree $n$ over $K$. Let $\mathcal{A}$ be a maximal order in $A$ defined over $X$. Let $s_{1}, \ldots, s_{N}$ be an admissible set of sections of $\mathcal{A}(d)$ and for any $\lambda \in k^{N}$, let $Y_{\lambda}$ be as above. There exists a nonempty open set $U \subset k^{N}$ such that, for any $\lambda \in U, Y_{\lambda}$ is an irreducible quasi-projective surface.

Before proving this theorem we recall, without proof, two easy lemmas.

Lemma 3.5. Let $\pi: Y \rightarrow X$ be a flat dominant morphism, with $X$ integral. Then $Y$ is reduced if and only if the generic fibre of $\pi$ is reduced.

Lemma 3.6. Let $\pi: Y \rightarrow X$ be a flat dominant morphism, with $X$ integral. Then $Y$ is irreducible if and only if the generic fibre of $\pi$ is irreducible.

Proof of Theorem 3.4. We set $\mathbb{A}_{k}^{N}=\operatorname{Spec}\left(k\left[t_{1}, \ldots, t_{N}\right]\right)$ and extend the base to $\widetilde{X}=X \times \mathbb{A}_{k}^{N}$. Let $\widetilde{A}$ and $\widetilde{\mathcal{L}}$ be the inverse images of $A$ and $\mathcal{L}$ under the projection $\pi: \widetilde{X} \rightarrow X$. Put $\widetilde{s}=t_{1} s_{1}+\cdots+t_{N} s_{N}$ and let $\widetilde{J}_{t}(T)$ be the characteristic ideal of $\widetilde{s}$ and $\widetilde{Y}$ the closed subscheme of $\operatorname{Spec}\left(\operatorname{Sym}\left(\widetilde{\mathcal{L}}^{-1}\right)\right)$ defined by $\widetilde{J}_{t}(T)$. Look at the diagram

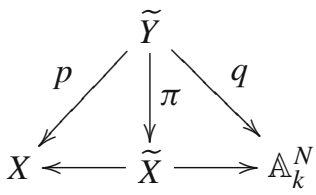

The map $\pi$ is clearly finite and flat and the two projections from $X \times \mathbb{A}_{k}^{N}$ are flat, hence $p$ and $q$ are flat. We set $\widetilde{Y}_{K}=\widetilde{Y} \times_{X} \operatorname{Spec}(K)$ and $q_{K}: \widetilde{Y}_{K} \rightarrow \mathbb{A}_{K}^{N}$ the restriction of $q$ to $\widetilde{Y}_{K}$. We first note that, by the choice of $s_{N}$ made above, the 
fibre $q_{K}^{-1}(0, \ldots, 0,1)$ is integral. By Theorem 9.7.7 of [5], to prove the theorem it suffices to show that the geometric generic fibre of $q$ is integral. Let $\Omega$ be an algebraic closure of $k\left(t_{1}, \ldots, t_{N}\right), \widetilde{Y}_{\Omega}=\widetilde{Y} \times_{\mathbb{A}_{k}^{N}} \operatorname{Spec}(\Omega)$ the generic fibre of $q$, $\widetilde{X}_{\Omega}=X \times_{k} \Omega$ and $\pi_{\Omega}: \widetilde{Y}_{\Omega} \rightarrow \widetilde{X}_{\Omega}$ the extension of $\pi$. Let $S$ be the integral closure of $k\left[t_{1}, \ldots, t_{N}\right]$ in $\Omega$ and $\Lambda=K \otimes_{k} S$. We set $\widetilde{Y}_{\Lambda}=\widetilde{Y} \times \widetilde{X} \operatorname{Spec}(\Lambda)$, $\widetilde{X}_{\Lambda}=\operatorname{Spec}(\Lambda)$ and $\pi_{\Lambda}: \widetilde{Y}_{\Lambda} \rightarrow \widetilde{X}_{\Lambda}$ the extension of $\pi$. Assume that $\widetilde{Y}_{\Omega}$ is not integral. Since $\pi_{\Omega}$ is flat, by 3.5 and 3.6 the generic fibre of $\pi_{\Omega}$ is not integral. But $\pi_{\Lambda}$ is also flat and has the same generic fibre as $\pi_{\Omega}$, hence, again by 3.5 and $3.5, \widetilde{Y}_{\Lambda}$ is not integral. The characteristic polynomial $P_{\tilde{s} / f}(T) \in K\left[t_{1}, \ldots, t_{N}\right]$ that generates $\widetilde{J}_{t}(T)$ over a suitable open set of $X$ is clearly separable over $K\left(t_{1}, \ldots, t_{N}\right)$, hence $\widetilde{Y}_{\Lambda}$ is reduced by Lemma 3.5. If $\widetilde{Y}_{\Lambda}$ is not integral, being reduced it has more than one component and since $\pi_{\Lambda}$ is finite and flat, each component maps surjectively onto $\widetilde{X}_{\Lambda}$ and hence no fibre is integral. Let $z$ be a point of $\widetilde{X}_{\Lambda}$ over the point $(0, \ldots, 0,1)$ of $\mathbb{A}_{K}^{N}$. Specializing at $z$ we get a contradiction with the irreducibility of $\pi_{\Lambda}^{-1}(0, \ldots, 0,1)=\operatorname{Spec}(K) \times_{X} Y_{(0, \ldots, 0,1)}$.

Corollary 3.7. Let $U$ be as in 3.4. For any $\lambda \in W$ the field $k\left(Y_{\lambda}\right)$ splits $A$.

Proof. By construction the field $k\left(Y_{\lambda}\right)$ is a maximal subfield of $A$.

We now assume that $\mathcal{A}$ is an Azumaya algebra over $X$ and show how to construct a smooth splitting, dealing first with the quasiprojective case in characteristic zero.

Proposition 3.8. Assume that $\mathcal{A}$ is an Azumaya algebra over $X$. The dimension of $\operatorname{Sing}(\widetilde{Y})$ is at most $N-1$.

Proof. We try to determine the singularities of $\widetilde{Y}$ using the following lemma.

Lemma 3.9. Let $f: Z \rightarrow X$ be a flat map of schemes. Suppose that $X$ is regular. If $z \in Z$ is a singular point of $Z$, then $z$ is a singularity of its fibre $f^{-1}(f(z))$.

Proof. Let $C$ be the local ring of $Z$ at $z$ and $A$ be the local ring of $f(z)$. By assumption the maximal ideal of $A$ is generated by a regular sequence $\left(x_{1}, \ldots, x_{m}\right)$. Since $f$ is flat, $C$ is faithfully flat over $A$ and this sequence is still regular as a sequence in $C$. If $z$ is not a singular point of its fibre, then $C /\left(x_{1}, \ldots, x_{m}\right)$ is regular and hence its maximal ideal is generated by a regular sequence $\left(\bar{y}_{1}, \ldots, \bar{y}_{r}\right)$. This implies that the maximal ideal of $C$ is generated by the regular sequence $\left(x_{1}, \ldots, x_{m}, y_{1}, \ldots, y_{r}\right)$, hence $C$ is regular.

By 3.9 the singularities of $\widetilde{Y}$ are contained in the union of the singularities of the fibres of $p$.

Lemma 3.10. For any $x \in X$ the singular locus of the fibre $p^{-1}(x)$ of $p$ has codimension 3 in $p^{-1}(x)$.

Proof. Let $k(x)$ be the residue field of $x \in X, \Omega$ its algebraic closure and $F_{x}$ the fibre of $p$ at $x$. The geometric fibre $\mathcal{A}(\bar{x})$ of $\mathcal{A}$ at $x$ is a matrix algebra $M_{n}(\Omega)$ and

$$
F_{\bar{x}}=\operatorname{Spec}\left(\Omega\left[t_{1}, \ldots, t_{N}\right][T] /\left(P_{x}(T)\right)\right),
$$


where $P_{x}(T)$ is the characteristic polynomial of $\bar{s}=\left(t_{1} s_{1}(x)+\cdots+t_{N} s_{N}(x)\right) /$ $f(x)$ for some generator $f$ of $\left.\mathcal{L}\right|_{U}, U$ a neighbourhood of $x$. Since the sections $s_{i}(x) / f(x)$ generate $M_{n}(\Omega)$ over $\Omega$, by a linear change of coordinates we may assume that $\bar{s}=t_{1} e_{1}+\cdots+t_{m} e_{m}$ where $m=n^{2}$ and $\left\{e_{1}, \ldots, e_{m}\right\}$ form a basis of $M_{n}(\Omega)$. Then

$$
F_{\bar{x}}=Y_{n} \times \operatorname{Spec}\left(\Omega\left[t_{m+1}, \ldots, t_{N}\right]\right) .
$$

We proved that $\operatorname{Sing}\left(Y_{n}\right)$ has codimension 3, hence the same holds for $\operatorname{Sing}\left(F_{\bar{x}}\right)$ and for $\operatorname{Sing}\left(F_{x}\right)$.

Theorem 3.11. The dimension of $\operatorname{Sing}(\widetilde{Y})$ is at most $N-1$.

Proof. For every $x \in X$ the fibre $F_{x}$ of $p$ is a finite cover of $\mathbb{A}_{k}^{N}$ and hence the dimension of $F_{X}$ is $N$. Let $\operatorname{Sing}(\widetilde{Y})$ be the singular locus of $\widetilde{Y}$. By 3.9, for every $x \in X$, the fibre at $x$ of $\left.p\right|_{\operatorname{Sing}(\widetilde{Y})}: \operatorname{Sing}(\widetilde{Y}) \rightarrow X$ is contained in the singular locus of $F_{X}$ and has therefore dimension at most $N-3$. Since $X$ is 2-dimensional, the dimension of $\operatorname{Sing}(\widetilde{Y})$ is at most $N-1$.

\section{Smooth splitting in characteristic zero}

Theorem 4.1. Let $k$ be an algebraically closed field of characteristic $0, X$ a smooth quasi-projective irreducible surface over $k, K=k(X)$ the field of rational functions of $X$. Let $\mathcal{A}$ be an Azumaya algebra over $X$ and $s_{1}, \ldots, s_{N}$ an admissible set of sections of $\mathcal{A}(d)$ as defined in Sect.3. For any $\lambda \in k^{N}$ let $Y_{\lambda}$ be the surface associated to the section $\lambda_{1} s_{1}+\cdots+\lambda_{N} s_{N}$. There exists a nonempty open set $V \subset k^{N}$ such that for any $\lambda \in V, Y_{\lambda}$ is a smooth integral quasi-projective surface. Further, the pull-back $\pi_{\lambda}^{*} \mathcal{A}$ is trivial in $\operatorname{Br}\left(Y_{\lambda}\right)$.

Proof. Look at $q: \widetilde{Y} \rightarrow \mathbb{A}_{k}^{N}$. Since by 3.11 $\operatorname{Sing}(\widetilde{Y})$ is at most $(N-1)$-dimensional, its image $q(\operatorname{Sing}(\tilde{Y}))$ is contained in a proper closed subset of $\mathbb{A}_{k}^{N}$. Choose an open set $W \subset \mathbb{A}_{k}^{N}$ which does not intersect $q(\operatorname{Sing}(\tilde{Y}))$ and let $\widetilde{W}=q^{-1}(W) \cap \tilde{Y}$. We now have a map $q: \widetilde{W} \rightarrow W$ of smooth varieties. This map is clearly flat and surjective and therefore, if $k$ is of characteristic zero, it is generically smooth (see [6], Chap. III, Corollary 10.7). By definition of generic smoothness there exists a dense open set $U^{\prime} \subset \mathbb{A}_{k}^{N}$ such that $q^{-1}\left(U^{\prime}\right) \cap \widetilde{Y} \rightarrow U^{\prime}$ is smooth. Thus for any $\lambda \in U^{\prime}$ the fibre $Y_{\lambda}=q^{-1}(\lambda) \cap \tilde{Y}$ is smooth. By 3.4, if $\lambda \in U$ then $Y_{\lambda}$ is integral, hence for any $\lambda \in V=U \cap U^{\prime}$ the surface $Y_{\lambda}$ is smooth and integral. By 3.7 the field $k\left(Y_{\lambda}\right)$ splits $A$. But $Y_{\lambda}$ being smooth, the canonical map $\operatorname{Br}\left(Y_{\lambda}\right) \rightarrow \operatorname{Br}\left(k\left(Y_{\lambda}\right)\right)$ is injective and thus $\pi_{\lambda}^{*} \mathcal{A}$ is trivial in $\operatorname{Br}\left(Y_{\lambda}\right)$.

Remark. In positive characteristic Theorem 4.1 is not true for arbitrary sets of admissible sections. Let for instance $X$ be the affine plane $X=\operatorname{Spec}(k[u, v])$ (the affine line would also suffice) over a field of odd characteristic $p$ and $\mathcal{A}$ the trivial Azumaya algebra $M_{2}\left(\mathcal{O}_{X}\right)$ over $X$. Then $\mathcal{A}$ is generated by its global sections 


$$
s_{1}=\left(\begin{array}{ll}
1 & u^{p} \\
0 & 0
\end{array}\right), \quad s_{2}=\left(\begin{array}{ll}
0 & 1 \\
0 & 0
\end{array}\right), \quad s_{3}=\left(\begin{array}{ll}
0 & 0 \\
1 & 0
\end{array}\right), \quad s_{4}=\left(\begin{array}{ll}
1 & u^{p} \\
1 & 1
\end{array}\right),
$$

and the generic splitting that we denoted $\widetilde{Y}$ is the spectrum of

$$
S=k\left[u, v, t_{1}, t_{2}, t_{3}, t_{4}\right][T] /(P(T))
$$

where the determinant $P(T)$ of $T \cdot \mathrm{I}_{2}-\left(t_{1} s_{1}+t_{2} s_{2}+t_{3} s_{3}+t_{4} s_{4}\right)$ is

$$
T^{2}-\left(t_{1}+2 t_{4}\right) T+t_{4}\left(t_{1}+t_{4}\right)-\left(t_{3}+t_{4}\right)\left(t_{2}+t_{4} u^{p}\right) .
$$

The algebra $S$ is smooth over $k$ if and only if $P, P^{\prime}, \partial P / \partial u$ and $\partial P / \partial v$ have no common zero over the algebraic closure of $k\left(t_{1}, t_{2}, t_{3}, t_{4}\right)$. But in fact, they are easily seen to be solvable with respect to $u$ provided $\left(t_{3}+t_{4}\right) t_{4} \neq 0$.

Still, the theorem is true in any characteristic if we choose more accurately the sections $s_{1}, \ldots, s_{N}$.

\section{Smooth splitting in arbitrary characteristic}

Lemma 5.1. Let $X \subset \mathbb{P}_{k}^{n}$ be a quasiprojective variety and let $\mathcal{F}$ be a coherent sheaf on $X$ generated by global sections $s_{1}, \ldots, s_{N}$. Let $V=H^{0}\left(X, \mathcal{O}_{X}(1)\right)=k x_{0}+$ $\cdots+k x_{n}$ where $x_{0}, \ldots, x_{n}$ are the projective coordinates on $X$. Let $W \subseteq H^{0}(X, \mathcal{F})$ be the $k$-space generated by $s_{1}, \ldots, s_{N}$. We denote by $m_{x}$ the maximal ideal of the local ring of any closed point $x$ of $X$.

(a) For any $x \in X(k)$ the canonical map

$$
V \rightarrow H^{0}\left(X, \mathcal{O}_{X}(1) \otimes \mathcal{O}_{X} \mathcal{O}_{X, x} / m_{x}^{2}\right)
$$

is surjective.

(b) For any $x \in X(k)$ the canonical map

$$
V \otimes_{k} W \rightarrow H^{0}\left(X, \mathcal{F}(1) \otimes_{\mathcal{O}_{X}} \mathcal{O}_{X, x} / m_{x}^{2}\right)
$$

is surjective.

Proof. The second assertion immediately follows from the first one. As to the first one, let $x \in \mathbb{P}_{k}^{n}$ be any closed point of $X$. It will be defined by the vanishing of $n$ linear forms, which we may assume to be $x_{1}, \ldots, x_{n}$. Then $m_{x}$ is the ideal of $\mathcal{O}_{X, x}$ generated by $x_{1} / x_{0}, \ldots, x_{n} / x_{0}$ and

$$
\mathcal{O}_{X, x} / m_{x}^{2}=k+k \overline{\left(x_{1} / x_{0}\right)}+\cdots+k \overline{\left(x_{n} / x_{0}\right)}
$$

where the bar denotes the class modulo $m_{x}^{2}$. We thus have

$$
H^{0}\left(\mathcal{O}_{X}(1) \otimes_{\mathcal{O}_{X}} \mathcal{O}_{X, x} / m_{x}^{2}\right)=k \bar{x}_{0}+\cdots+k \bar{x}_{n}
$$

which proves the assertion. 
Let $X$ be an irreducible quasiprojective smooth surface over $k$ and $\mathcal{A}$ an Azumaya algebra of degree $n$ over $X$. We assume here that, by the lemma we just proved, we have chosen the line bundle $\mathcal{L}$ such that the global sections $s_{1}, \ldots, s_{N}$ generate

$$
H^{0}\left(X, \mathcal{A} \otimes \mathcal{O}_{x} \mathcal{L} \otimes \mathcal{O}_{X} \mathcal{O}_{X, x} / m_{x}^{2}\right)
$$

as a vector space over $k$ for every closed point $x \in X(k)$.

We still assume that $s_{N}=\sigma g$ with $g \neq 0$ a section of $\mathcal{L}$ and $\sigma$ as in Lemma 3.1. Let $p: \widetilde{Y} \rightarrow X$ and $\widetilde{Y} \rightarrow \mathbb{A}_{k}^{N}$ be as above. We study under which conditions the fibre of $Y_{\lambda} \rightarrow X$ at $x \in X(k)$ is singular. We fix an $x$ in $X(k)$ and set $R=\mathcal{O}_{X, x}$, $m=m_{x}$ and $\bar{R}=R / m^{2}$. Reduction modulo $m^{2}$ will systematically be denoted by a bar. Let $\xi, \eta$ be generators of $m$. Then, $\bar{R}=k[\xi, \eta]$ with $\xi^{2}=\xi \eta=\eta^{2}=0$. We choose an isomorphism $\mathcal{A}(\operatorname{Spec}(R)) \otimes_{R} \bar{R} \simeq M_{n}(\bar{R})$, and a local section $f \neq 0$ of $\mathcal{L}$ defining an isomorphism $\mathcal{L}(\operatorname{Spec}(R)) \rightarrow R$. Consider the composition of $k$-linear maps

$$
\begin{aligned}
\varphi: & k^{N} \rightarrow H^{0}\left(X, \mathcal{A} \otimes \mathcal{O}_{X} \mathcal{L}\right) \rightarrow \mathcal{A}(\operatorname{Spec}(R)) \otimes_{R} \mathcal{L}(\operatorname{Spec}(R)) \rightarrow \mathcal{A}(\operatorname{Spec}(R)) \\
& \rightarrow M_{n}(\bar{R})
\end{aligned}
$$

mapping $\lambda$ to the image of $s_{\lambda} / f$.

We write every element $\bar{a}$ of $M_{n}(\bar{R})$ as $\bar{a}=\alpha+\beta \xi+\gamma \eta$ with $\alpha, \beta$ and $\gamma \in M_{n}(k)$. Suppose now that $s_{\lambda} / f=a \in \mathcal{A}(R)$ is the local section corresponding to $\lambda \in \mathbb{A}_{k}^{N}$ and $\bar{a}$ its image in $M_{n}(\bar{R})$. The reduction modulo $m^{2}$ of the local affine algebra of $\widetilde{Y}$ at $(x, \lambda)$ is

$$
\bar{R}[T] / \bar{P}_{\lambda}(T)
$$

where

$$
P(T)=T^{n}+a_{1} T^{n-1}+\cdots+a_{n-1} T+a_{n}
$$

is the characteristic polynomial of $a$. We denote its reduction modulo $m$ by $\overline{\bar{P}}(T)$. We introduce the set of matrices

$$
S(x)=\left\{\bar{a} \in M_{n}(\bar{R}) \mid \exists \lambda \in k^{N} \text { s.t. } \varphi(\lambda)=\bar{a} \text { and } Y_{\lambda} \text { is singular }\right\}
$$

and set $\widetilde{S}(x)=\varphi^{-1}(S(x))$. Observe that $\widetilde{S}(x)$ does not depend on the choice of the local section $f$ because if $\bar{a} \in S(x)$ then $\bar{a} u \in S(x)$ for any unit $u$ of $\bar{R}$.

Proposition 5.2. The codimension of $S(x)$ in $M_{n}(\bar{R})$ is as least 3 .

Proof. We consider more cases than what is really necessary because we want to prepare the way for the Galois splitting in the next section.

Fix a point $y=(x, \mu) \in Y_{\lambda}$ in the fibre of $x$, where $\mu$ is a root of $\overline{\bar{P}}(T) \in k[T]$. The fibre of $p: Y_{\lambda} \rightarrow X$ at $x$ is singular at $y$ if and only if the derivatives $\frac{\partial \bar{P}}{\partial T}, \frac{\partial \bar{P}}{\partial \xi}, \frac{\partial \bar{P}}{\partial \eta}$ vanish at $y=(x, \mu)$. To see what this means we write $\bar{a}=\alpha+\xi \beta+\eta \gamma$ with $\alpha, \beta$ and $\gamma$ in $M_{n}(k)$. If $\mu$ is a simple root, then $\frac{\partial \bar{P}}{\partial T} \neq 0$ at $(x, \mu)$ and $(x, \mu)$ is a smooth point of $Y_{\lambda}$. Assume therefore that $\alpha$ has at least two identical eigenvalues. The set 
of all matrices $\alpha \in M_{n}(k)$ with at most $n-3$ different eigenvalue has codimension 3 , so we only have to deal with the cases in which $\alpha$ has $n-1$ or $n-2$ distinct eigenvalues. This is the same as saying that $\alpha$ is conjugated to a matrix

$$
\left(\begin{array}{ll}
J_{i} & 0 \\
0 & D
\end{array}\right)
$$

where $D$ is a diagonal matrix with distinct eigenvalues, different from $\mu$ for $1 \leq$ $i \leq 5$ and distinct from $\mu$ and $v$ for $6 \leq i \leq 8$ and $\mu \neq v$ and $J_{i}$ is one of the following matrices

$$
\begin{gathered}
J_{1}=\left(\begin{array}{cc}
\mu & 0 \\
0 & \mu
\end{array}\right), J_{2}=\left(\begin{array}{ll}
\mu & 1 \\
0 & \mu
\end{array}\right), \\
J_{3}=\left(\begin{array}{lll}
\mu & 0 & 0 \\
0 & \mu & 0 \\
0 & 0 & \mu
\end{array}\right), J_{4}=\left(\begin{array}{ccc}
\mu & 1 & 0 \\
0 & \mu & 0 \\
0 & 0 & \mu
\end{array}\right), J_{5}=\left(\begin{array}{ccc}
\mu & 1 & 0 \\
0 & \mu & 1 \\
0 & 0 & \mu
\end{array}\right), \\
J_{6}=\left(\begin{array}{llll}
\mu & 0 & 0 & 0 \\
0 & \mu & 0 & 0 \\
0 & 0 & v & 0 \\
0 & 0 & 0 & v
\end{array}\right), J_{7}=\left(\begin{array}{llll}
\mu & 1 & 0 & 0 \\
0 & \mu & 0 & 0 \\
0 & 0 & v & 0 \\
0 & 0 & 0 & v
\end{array}\right), J_{8}=\left(\begin{array}{cccc}
\mu & 1 & 0 & 0 \\
0 & \mu & 0 & 0 \\
0 & 0 & v & 1 \\
0 & 0 & 0 & v
\end{array}\right) .
\end{gathered}
$$

For $1 \leq i \leq 8$ let $M_{n}^{i}$ be the set of all matrices $\bar{a} \in M_{n}(\bar{R})$ for which $\alpha$ is of the form $\operatorname{diag}\left(J_{i}, D\right)$ and $\beta$ and $\gamma$ are arbitrary matrices in $M_{n}(k)$. These sets are open subsets of affine spaces, in particular they are irreducible. We denote by $\widehat{M}_{n}^{i}$ the $G l_{n}(k)$-orbit of $M_{n}^{i}$ and by $G_{i}$ the stabilizer of $M_{n}^{i}$ in $G l_{n}(k)$. Since $G l_{n}(k)$ is irreducible, all $\widehat{M}_{n}^{i}$,s are irreducible. From the formula

$$
\operatorname{dim}\left(\widehat{M}_{n}^{i}\right) \leq \operatorname{dim}\left(M_{n}^{i}\right)+\operatorname{dim}\left(G l_{n}(K)\right)-\operatorname{dim}\left(G_{i}\right)
$$

we first compute an upper bound for the dimension of each $\widehat{M}_{n}^{i}$.

Using that if $M \in M_{m}(k)$ is either a Jordan block or a diagonal matrix with distinct eigenvalues, then its stabilizer in $G l_{m}(k)$ has dimension $m$, together with a direct computation for $G_{4}$ we find $\operatorname{dim}\left(G_{1}\right) \geq n+2, \operatorname{dim}\left(G_{2}\right) \geq n, \operatorname{dim}\left(G_{3}\right) \geq n+6$, $\operatorname{dim}\left(G_{4}\right) \geq n+2, \operatorname{dim}\left(G_{5}\right) \geq n, \operatorname{dim}\left(G_{6}\right) \geq n+4, \operatorname{dim}\left(G_{7}\right) \geq n+2, \operatorname{dim}\left(G_{8}\right) \geq$ $n+2$.

On the other hand, $\operatorname{dim}\left(M_{n}^{i}\right)=2 n^{2}+n-1$ for $i=1,2$ and $2 n^{2}+n-2$ for $3 \leq i \leq 8$. Thus the codimension of $\widehat{M}_{n}^{2}$ is 1 , that of $\widehat{M}_{n}^{5}, \widehat{M}_{n}^{8}$ is 2 and the remaining ones have codimension $\geq 3$. hence we only have to consider the singularities arising from $\widehat{M}_{n}^{2}, \widehat{M}_{n}^{5}$, and $\widehat{M}_{n}^{8}$.

We shall show that if $\bar{a}=\alpha+\xi \beta+\eta \gamma$ is in $S(x) \cap \widehat{M}_{n}^{2}$, then $\beta$ and $\gamma$ must both belong to certain proper closed subsets of $M_{n}(k)$.

The point $(x, \mu)$ is singular if and only if both $\frac{\partial \bar{P}}{\partial \xi}$ and $\frac{\partial \bar{P}}{\partial \eta}$ vanish at $T=\mu$. To compute $\bar{P}(T)$ we can use the following lemma. 
Lemma 5.3. Let $A$ be a commutative ring, $I \subset A$ an ideal such that $I^{2}=(0)$, and $M \in M_{n}(A)$ a matrix of the form

$$
\left(\begin{array}{ll}
a & b \\
c & d
\end{array}\right)
$$

with a, $d$ square blocks and $b, c$ having entries in $I$. The characteristic polynomial of $M$ is $P_{M}(T)=P_{a}(T) P_{d}(T)$ where $P_{a}$ and $P_{d}$ are the characteristic polynomials of $a$ and $d$ respectively.

Proof. Since $P_{a}(T)$ is not a zero divisor, we can embed $A$ into $A\left[T, 1 / P_{a}(T)\right]$ and compute in this overring, using the fact that $M_{n}\left(A\left[T, 1 / P_{a}(T)\right]\right)$ contains $(T-a)^{-1}$. We have

$$
\begin{gathered}
\operatorname{det}\left(\begin{array}{ll}
T-a & -b \\
-c & T-d
\end{array}\right)=\operatorname{det}\left(\begin{array}{ll}
1 & 0 \\
c(T-a)^{-1} & 1
\end{array}\right) \operatorname{det}\left(\begin{array}{ll}
T-a & -b \\
-c & T-d
\end{array}\right) \\
\quad=\operatorname{det}\left(\begin{array}{ll}
T-a & -b \\
-0 & -c(T-a)^{-1} b+T-d
\end{array}\right)=\operatorname{det}\left(\mathrm{T}_{\mathrm{a}}\right) \operatorname{det}\left(\mathrm{T}_{\mathrm{d}}\right)
\end{gathered}
$$

because $c(T-a)^{-1} b=0$.

We now complete the proof of 5.2. Using 5.3 we see that, if $\bar{a}$ is in $M_{n}^{2}, \beta=\left(\beta_{i, j}\right)$ and $\gamma=\left(\gamma_{i, j}\right)$, then

$$
\left.\left(\frac{\partial \bar{P}}{\partial \xi}, \frac{\partial \bar{P}}{\partial \eta}\right)\right|_{\substack{T=\mu \\ \xi, \eta)=(0,0)}}=\left(-\beta_{2,1},-\gamma_{2,1}\right) P_{D}(\mu)
$$

where $P_{D}(T)$ - the characteristic polynomial of $D$-does not vanish at $\mu$. Hence, the point $(x, \mu)$ is singular if and only if

$$
\beta_{2,1}=0 \text { and } \gamma_{2,1}=0 .
$$

This shows that $S(x) \cap M_{n}^{2}$ is of codimension 2 in $M_{n}^{2}$, hence of codimension at least 3 in $M_{n}(\bar{R})$. Since $G_{2}$ also stabilizes $S(x) \cap M_{n}^{2}$, the codimension of its orbit $S(x) \cap \widehat{M}_{n}^{2}$ is at least 3 .

In the remaining two cases the codimension of $\widehat{M}_{n}^{i}$ is 2 and, as we have seen, the set $\widehat{M}_{n}^{i}$ is irreducible. Since the set of matrices $\bar{a} \in M_{n}(\bar{R})$ for which $(x, \mu)$ is a smooth point is an open set, to show that $S(x) \cap \widehat{M}_{n}^{i}$ is of codimension $\geq 3$ it suffices to show that $\widehat{M}_{n}^{i}$ contains a matrix for which the fibre of $x$ consists of smooth points. A direct computation shows that if

$$
A=\left(\begin{array}{lll}
0 & 1 & 0 \\
\xi & 0 & 1 \\
\eta & 0 & 0
\end{array}\right) \quad \text { and } B=\left(\begin{array}{cccc}
0 & 1 & 0 & 0 \\
\xi & 0 & 0 & 0 \\
0 & 0 & 1 & 1 \\
0 & 0 & \eta & 1
\end{array}\right)
$$

then for a diagonal with distinct eigenvalues different from 0 and $1, \operatorname{diag}(A, D) \in$ $\widehat{M}_{n}^{5} \backslash S(x)$ and $\operatorname{diag}(B, D) \in \widehat{M}_{n}^{8} \backslash S(x)$.

This finishes the proof of 5.2. 
We now show the existence of smooth splittings.

Theorem 5.4. Let $X$ be an irreducible quasiprojective smooth surface over $k$ and $\mathcal{A}$ an Azumaya algebra of degree $n$ over X. Assume (5.1) that we have chosen the line bundle $\mathcal{L}$ such that the global sections $s_{1}, \ldots, s_{N}$ generate

$$
H^{0}\left(X, \mathcal{A} \otimes \mathcal{O}_{x} \mathcal{L} \otimes \mathcal{O}_{X} \mathcal{O}_{X, x} / m_{x}^{2}\right)
$$

for every closed point $x \in X(k)$. Assume also that $s_{N}=\sigma g$ with $g \neq 0$ a section of $\mathcal{L}$ and $\sigma$ are as in Lemma 3.1. Then there exists an open dense set $U \subset k^{N}$ such that, for any $\lambda \in U$ the surface $Y_{\lambda}$ is a smooth irreducible finite cover of $X$ and splits $\mathcal{A}$.

Proof. It only remains to prove smoothness for $\lambda$ varying in a suitable open set $U$. Since, by the choice of $s_{1}, \ldots, s_{N}$, the linear map $\varphi$ is surjective, $\widetilde{S}(x)$ is a closed set of codimension $\geq 3$ in $k^{N}$. Let $\widetilde{S}$ be the union of all $\widetilde{S}(x)$ for $x$ running over $X(k)$.

Let now $\Sigma \subset \widetilde{Y}(k)$ be the closed set of points of $\widetilde{Y}(k)$ at which the map $q: \widetilde{Y} \rightarrow \mathbb{A}_{k}^{N}$ is not smooth. Since $q$ is flat, being smooth is the same as having smooth fibres and therefore its image $q(\Sigma)$ in $k^{N}$ is $\widetilde{S}$, which is closed because $q$ is a projective map. We want to show that $\widetilde{S}$ is a proper closed subset of $k^{N}$. For any $x \in X(k)$ the closed set $\Sigma(x):=\pi^{-1}\left(x \times k^{N}\right) \cap \Sigma$ is mapped by $q$ onto $\widetilde{S}(x)$, which has codimension $\geq 3$ in $k^{N}$. Since $q$ is a flat surjective map, $\Sigma(x)$ has codimension $\geq 3$ in $\pi^{-1}\left(x \times k^{N}\right)$, hence dimension at most $N-3$. Since $X$ is two-dimensional the dimension of $\Sigma$ is at most $N-1$. This shows that its image $\widetilde{S}$ in $k^{N}$ is a proper closed subset of $k^{N}$. From this we conclude that for a general $\lambda \in k^{N}$ the surface $Y_{\lambda}$ is smooth.

\section{Smooth finite Galois splitting of Azumaya algebras}

We now construct, for any $\lambda \in k^{N}$, a Galois covering $Z_{\lambda}$ of $X$ with group $G=\mathcal{S}_{n}$, such that $X=Z_{\lambda} / G$. Notice that, in general, even if $Y_{\lambda}$ is smooth its Galois closure may be singular. Therefore, in order to have $Y$ and $Z$ smooth we must construct both at the same time. We achieve this by globalizing the construction of the universal splitting algebra of a monic polynomial, which we now recall.

Let $R$ be a commutative ring and $P(T)=T^{n}+b_{1} T^{n-1}+\cdots+b_{n}$ a monic polynomial with coefficients in $R$. For $1 \leq i \leq n$ let $\sigma_{i}$ be the $i$-th elementary symmetric function in the $n$ variables $T_{1}, \ldots, T_{n}$. The universal splitting algebra of $P(T)$ is the quotient $S$ of the polynomial algebra $R\left[T_{1}, \ldots, T_{n}\right]$ by the ideal $I$ generated by the elements

$$
\sigma_{i}\left(T_{1}, \ldots, T_{n}\right)-(-1)^{i} b_{i}, \quad 1 \leq i \leq n .
$$

We denote by $\tau_{1}, \ldots, \tau_{n}$ the classes modulo $I$ of $T_{1}, \ldots, T_{n}$. We clearly have

$$
P(T)=\left(T-\tau_{1}\right) \cdots\left(T-\tau_{n}\right) .
$$

The symmetric group $\mathcal{S}_{n}$ operates on $S$ by permuting $\tau_{1}, \ldots, \tau_{n}$.

We will use the following properties of $S$. (For more details and proofs see [1] or [3]). 
$P 1$. The construction of $S$ commutes with scalar extensions ([3], 1.9).

$P 2$. As an $R$-module $S$ is free of rank $n$ ! ([3], 1.10).

$P 3$. For any commutative $R$-algebra $A$ and any $n$-tuple $\left(a_{1}, \ldots, a_{n}\right)$ of elements of $A$ such that $p(T)=\left(T-a_{1}\right) \cdots\left(T-a_{n}\right)$ in $A[T]$ there is a unique $R$-homomorphism $\varphi: S \rightarrow A$ such that $\varphi\left(\tau_{i}\right)=a_{i}([3], 1.3)$.

$P 4$. The subalgebra $R\left[\tau_{n}\right]$ of $S$ is isomorphic to $R[T] /(P(T))$ and $S$ is the universal splitting algebra of $P(T) /\left(T-\tau_{n}\right)$ over $R\left[\tau_{n}\right]([3], 1.8)$.

$P$ 5. If the discriminant of $P(T)$ is a regular element of $R$, then $S^{\mathcal{S}_{n}}=R$ ([3], 2.2).

$P 6$. If $R$ is a field and $P(T)$ is separable with Galois group $\mathcal{S}_{n}$, then $S$ is a Galois extension of $R$ with Galois group $\mathcal{S}_{n}$.

We now construct $Z_{\lambda}$. Let $\mathcal{L}$ be a very ample line bundle such that $\mathcal{A} \otimes_{\mathcal{O}_{X}} \mathcal{L}$ is generated by global sections $s_{1}, \ldots, s_{N}$ and assume that $s_{N}=\sigma g$ with $g \neq 0$ a global section of $\mathcal{L}$ and $\sigma$ as in Lemma 3.1. Let $U \subset X$ be an affine open set for which $\left.\mathcal{L}\right|_{U}$ is isomorphic to $\mathcal{O}_{U} f$ for some section $f$ on $U$. We set, as in Sect. 3, $s=\lambda_{1} s_{1}+\cdots \lambda_{N} s_{N}$. Let $P_{f, U}(T)=T^{n}+b_{1} T^{n-1}+\cdots+b_{n}$ be the characteristic polynomial of $s / f \in \mathcal{A}(U)$. We choose $n$ isomorphic copies $\mathcal{L}_{1}, \ldots, \mathcal{L}_{n}$ of $\mathcal{L}$ and for each $i, f_{i}=f$ the generator of $\left.\mathcal{L}_{i}\right|_{U}$. Consider

$$
\mathcal{T}=\operatorname{Sym}\left(\mathcal{L}_{1}^{-1} \oplus \cdots \oplus \mathcal{L}_{n}^{-1}\right)
$$

Writing $f_{i}^{-1} f_{j}^{-1}$ instead of $f_{i}^{-1} \otimes \mathcal{O}_{U} f_{j}^{-1}$ we shall write the restriction of $\mathcal{T}$ to $U$ simply as

$$
\bigoplus \mathcal{O}_{U} f_{1}^{-i_{1}} \cdots f_{n}^{-i_{n}}
$$

Note that $\mathcal{O}_{U}\left[T_{1}, \ldots, T_{n}\right]$ is isomorphic to $\left.\mathcal{T}\right|_{U}$ under $T_{i} \mapsto f_{i}^{-1}$.

We define $\left.\mathcal{J}_{f, U} \subset \mathcal{T}\right|_{U}$ as the ideal generated by

$$
\sigma_{i}\left(f_{1}^{-1}, \ldots, f_{n}^{-1}\right)-(-1)^{i} b_{i}, 1 \leq i \leq n .
$$

It corresponds in the polynomial algebra to the ideal generated by

$$
F_{i}=\sigma_{i}\left(T_{1}, \ldots, T_{n}\right)-(-1)^{i} b_{i}, 1 \leq i \leq n
$$

which defines the universal splitting algebra of $P_{f, U}(T)$. As in the preceding section, it is easy to check that these ideals do not depend on the choice of $f$ and can therefore be patched over the various $U$ 's to obtain a global ideal $\mathcal{J}_{\lambda} \subset \mathcal{T}$.

Let $Z_{\lambda}$ be the closed subscheme of $\operatorname{Spec}(\mathcal{T})$ defined by $\mathcal{J}_{\lambda}$.

Proposition 6.1. Assume that $\lambda \in k^{N}$ has been chosen such that $P_{f, U}(T)=P(T)$ is separable and irreducible over $K$. The symmetric group $\mathcal{S}_{n}$ acts on $Z_{\lambda}$ via its obvious action on $\mathcal{T}$. The quotient $Z_{\lambda} / \mathcal{S}_{n}$ coincides with $X$ and $Y_{\lambda}$ coincides with the quotient $Z_{\lambda} / \mathcal{S}_{n-1}$, where $\mathcal{S}_{n-1}$ is the isotropy group of 1 .

Proof. It suffices to deal with the affine case, when $S$ is the universal splitting algebra of $P(T)$ over $R=k[U]$ and show that $S^{\mathcal{S}_{n}}=R$ and $S^{\mathcal{S}_{n-1}}=R[T] /(P(T))$. Since $P(T)$ is separable over $K$ the first assertion follows from property P6 and the second from properties P3 and P6. 
Theorem 6.2. There exists a nonempty open set $U \subset k^{N}$ such that, for any $\lambda \in U$, $Z_{\lambda}$ is an irreducible quasi-projective surface. The natural map $\pi_{\lambda}: Z_{\lambda} \rightarrow X$ is a ramified Galois cover with group $\mathcal{S}_{n}$ and splits $\mathcal{A}$.

Proof. The splitting property follows from Proposition 6.1 because $Z_{\lambda} / \mathcal{S}_{n-1}=Y_{\lambda}$ which splits $\mathcal{A}$. It remains to prove that for a general $\lambda$ the fibre $Z_{\lambda}$ is irreducible. We extend the base to $\widetilde{X}=X \times \mathbb{A}_{k}^{N}$ where $\mathbb{A}_{k}^{N}=\operatorname{Spec}\left(k\left[t_{1}, \ldots, t_{N}\right]\right)$ and define $\widetilde{\mathcal{A}}, \widetilde{\mathcal{L}}$ and $\widetilde{\mathcal{L}}_{i}$ for $1 \leq i \leq n$ as the inverse images of $\mathcal{A}, \mathcal{L}$ and the $\mathcal{L}_{i}$ 's under the projection $\pi: \widetilde{X} \rightarrow X$. Repeating the construction of $\mathcal{J}_{\lambda}$ we obtain an ideal $\mathcal{J}_{t}$, where $t=\left(t_{1}, \ldots, t_{N}\right)$, which specializes to $\mathcal{J}_{\lambda}$ when we specialize $t$ to $\lambda$. The scheme $\widetilde{Z}$ is the closed subscheme of

$$
\operatorname{Spec}(\widetilde{\mathcal{T}})=\operatorname{Spec}\left(\operatorname{Sym}\left({\widetilde{\mathcal{L}_{1}}}^{-1} \oplus \cdots \oplus{\widetilde{\mathcal{L}_{n}}}^{-1}\right)\right)
$$

defined by $\mathcal{J}_{t}$.

Look at the diagram

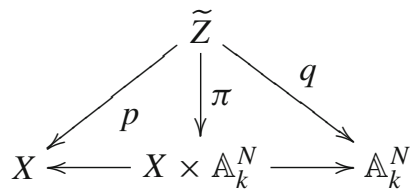

The map $\pi$ is clearly finite and flat and the two projections from $X \times \mathbb{A}_{k}^{N}$ are flat, hence $p$ and $q$ are flat. As in the previous section we set $\widetilde{Z}_{K}=\widetilde{Z} \times_{X} \operatorname{Spec}(K)$ and $q_{K}: \widetilde{Z}_{K} \rightarrow \mathbb{A}_{K}^{N}$ the restriction of $q$ to $\widetilde{Z}_{K}$. We first note that, by the choice of $s_{N}$ made above, the fibre $q_{K}^{-1}(0, \ldots, 0,1)$ is integral. In fact, by construction, its coordinate algebra is the universal splitting algebra of the characteristic polynomial $P_{S_{N} / f}(T)$ of $s_{N} / f$. Since the Galois group of $P_{S_{N} / f}(T)$ is $\mathcal{S}_{n}$, its universal splitting algebra, by property $\mathrm{P} 6$, is a field. We can now complete the proof exactly as we did in the proof of Theorem 3.4. By Theorem 9.7.7 of [5], it suffices to show that the geometric generic fibre of $q$ is integral. Let $\Omega, S, \Lambda$ and $\widetilde{X}_{\Lambda}$ be as in Sect. 3 and define $\widetilde{Z}_{\Omega}, \widetilde{Z}_{\Lambda}, \pi_{\Omega}$ and $\pi_{\Lambda}$ as we did there for $\widetilde{Y}_{\Omega}$ and so on. The proof given in Sect. 3 goes through once we remark that the universal splitting algebra $\widetilde{Z}_{\Lambda}$ is reduced. This is a special case of the following lemma.

Lemma 6.3. Let $R$ be a domain, $K$ its field of fractions and $P(T) \in R[T]$ a monic polynomial. Assume that $P(T)$ is separable over $K$. Then the universal splitting algebra of $P(T)$ over $R$ is reduced.

Proof. Let $S$ be the universal splitting algebra of $P(T)$ over $R$. It is a free $R$-algebra of degree $n$ !. The construction of the universal splitting algebra commutes with scalar extensions (property P1), hence $S \otimes_{R} K$ is the splitting algebra of $P(T)$ over $K$. Since $P(T)$ is separable over $K$, it follows immediately from property $\mathrm{P} 4$ that $S \otimes_{R} K$ is étale over $K$, in particular reduced. By Lemma 3.5 $S$ is reduced too. 


\section{Smooth Galois splitting in characteristic zero}

Theorem 7.1. Assume that $k$ is of characteristic zero. There exists a nonempty open set $U \subset k^{N}$ such that, for any $\lambda \in U, Z_{\lambda}$ is a quasi-projective irreducible smooth Galois covering of $X$ with Galois group $\mathcal{S}_{n}$ which splits $\mathcal{A}$.

Proof. If $n=2$ then $U=k^{N}$ and for any $\lambda \in k^{N}, Z_{\lambda}=Y_{\lambda}$. We therefore assume that $n \geq 3$. In this case the proof is on similar lines as the proof of Theorem 3.11. By 2.12 the singularities of $\widetilde{Z}$ are contained in the union of the singularities of the fibers of $p$. Since, by Theorem 4.1, the singularities of the closed fibres of $p$ are at worst in codimension 3, we can argue exactly as in the proof of Theorem 3.12 and conclude that $q$ is generically smooth. The other assertion are given by Theorem 6.2.

\section{Smooth Galois splitting in arbitrary characteristic}

Theorem 8.1. Let $X$ be an irreducible quasiprojective smooth surface over $k$ and $\mathcal{A}$ an Azumaya algebra of degree $n$ over X. Assume (5.1) that we have chosen the line bundle $\mathcal{L}$ such that the global sections $s_{1}, \ldots, s_{N}$ generate

$$
H^{0}\left(X, \mathcal{A} \otimes \mathcal{O}_{x} \mathcal{L} \otimes \mathcal{O}_{X} \mathcal{O}_{X, x} / m_{x}^{2}\right)
$$

for every closed point $x \in X(k)$. Assume also that $s_{N}=\sigma g$ with $f \neq 0$ a section of $\mathcal{L}$ and $\sigma$ are as in Lemma 3.1. Then there exists an open dense set $U \subset k^{N}$ such that, for any $\lambda \in U$ the surface $Z_{\lambda}$ is a smooth irreducible finite Galois cover of $X$ with Galois group $\mathcal{S}_{n}$, and splits $\mathcal{A}$.

Only the smoothness of a general fibre needs to be proved.

Let $x$ be closed point of $X, \lambda \in k^{N}$, and

$$
\bar{P}(T)=T^{n}+\bar{a}_{1} T^{n-1}+\cdots+\bar{a}_{n}
$$

the characteristic polynomial of $\varphi(\lambda) \in M_{n}(\bar{R})$. We defined $F_{i}=\sigma_{i}\left(T_{1}, \ldots, T_{n}\right)-$ $(-1)^{i} \bar{a}_{i}$ where $\sigma_{i}$ is the $i$-th elementary symmetric function. We define $\sigma_{i, j}^{\prime}$ as the $i$-th elementary symmetric function in $T_{1}, \ldots, T_{j-1}, T_{j+1}, \ldots, T_{n}$ and set $\sigma_{0, j}^{\prime}=1$. Note that $\partial F_{i} / \partial T_{j}=\sigma_{i-1, j}^{\prime}$. Let $\left(\mu_{1}, \ldots, \mu_{n}\right)$ be the roots of $\overline{\bar{P}}(T)$ in some chosen order. Then $z=\left(x, \mu_{1}, \ldots, \mu_{n}\right)$ is a point of $Z_{\lambda}$. It is smooth if and only if the jacobian matrix

$$
J(z)=\frac{\partial\left(F_{1}, \ldots, F_{n}\right)}{\partial\left(T_{1}, \ldots, T_{n}, \xi, \eta\right)}=\left(\begin{array}{ccccc}
1 & \cdots & 1 & -\frac{\partial a_{1}}{\partial \xi} & -\frac{\partial a_{1}}{\partial \eta} \\
\sigma_{1,1}^{\prime} & \cdots & \sigma_{1, n}^{\prime} & \frac{\partial a_{2}}{\partial \xi} & \frac{\partial a_{2}}{\partial \eta} \\
\vdots & & \vdots & \vdots & \vdots \\
\sigma_{n-1,1}^{\prime} & \cdots & \sigma_{n-1, n}^{\prime} & (-1)^{n} \frac{\partial a_{n}}{\partial \xi} & (-1)^{n} \frac{\partial a_{n}}{\partial \eta}
\end{array}\right)
$$

evaluated at $z$ (we denote it by $J(z)$ ) has rank $n$. In this section $S(x)$ will denote the set of $\bar{a}=\alpha+\xi \beta+\eta \gamma \in M_{n}(\bar{R})$ for which the fibre of $x$ contains a singular point of $Z_{\lambda}$, which is the same as saying that the corresponding Jacobian matrix has rank less than $n$. 
Proposition 8.2. The codimension of $S(x)$ in $M_{n}(\bar{R})$ is at least 3 .

Proof. If $\mu_{1}, \ldots, \mu_{n}$ are all distinct, then the Jacobian $\left(\partial \sigma_{i} / \partial T_{j}\right)$ evaluated at the point $\left(\mu_{1}, \ldots, \mu_{n}\right)$ is invertible and hence $J(z)$ has rank $n$. Suppose now that $\alpha$ has a multiple eigenvalue. As in Sect. 3 we only have to consider matrices in $\widehat{M}_{n}^{2}$, $\widehat{M}_{n}^{5}$ and $\widehat{M}_{n}^{8}$.

Suppose first that $\bar{a}$ is in $M_{n}^{2}$. In this case $\alpha$ has two equal eigenvalues $\mu_{1}=\mu_{2}=\mu$. Consider the $(n-1) \times(n-1)$ submatrix $T=\left(\sigma_{i-1, j}^{\prime}\right)$ of $J(z)$, with $1 \leq i \leq n-1$ and $2 \leq j \leq n$, evaluated at $z$

By multiplying the first row of $J(z)$ by $\mu$ and substracting it from the second, then multiplying the second by $\mu$ and substracting it from the third, and so on, we transform $T$ into $T^{\prime}=\left(\partial s_{i} / \partial T_{j}\right), 1 \leq i \leq n-1,2 \leq j \leq n$, evaluated at $\left(\mu, \mu_{3}, \ldots, \mu_{n}\right)$ where $s_{i}$ is the $i$-th elementary symmetric function in the $n-1$ variables $T_{2}, \ldots, T_{n}$. Since $\mu, \mu_{3}, \ldots, \mu_{n}$ are all distinct $T^{\prime}$, is invertible. This proves that the columns of $J(z)$ from the second to the $n$-th are independent. By these row operations the last row of $J(z)$ becomes

$$
\left(0,0, \ldots, 0,(-1)^{n-1} \frac{\partial \bar{P}}{\partial \xi}(\mu),(-1)^{n-1} \frac{\partial \bar{P}}{\partial \eta}(\mu)\right)
$$

and therefore the rank of $J(z)$ will be $n$ if and only if

$$
\left(\frac{\partial \bar{P}}{\partial \xi}(\mu), \frac{\partial \bar{P}}{\partial \eta}(\mu)\right) \neq(0,0) .
$$

We already computed $\bar{P}(T)$ in 3 and found that its derivatives with respect to $\xi$ and $\eta$ both vanish for $\xi=\eta=0$ and $T=\mu$ if and only if

$$
\beta_{2,1}=0 \text { and } \gamma_{2,1}=0 \text {. }
$$

These two conditions show that the codimension of $\widehat{M}_{n}^{2} \cap S(x)$ is $\geq 3$. The case $n=4$ will illustrate what we said. The matrix $J(z)$ is

$$
\left(\begin{array}{cccccc}
1 & 1 & 1 & 1 & \frac{\partial \bar{a}_{1}}{\partial \xi} & \frac{\partial \bar{a}_{1}}{\partial \eta} \\
\mu+\mu_{3}+\mu_{4} & \mu+\mu_{3}+\mu_{4} & \mu+\mu+\mu_{4} & \mu+\mu+\mu_{3} & -\frac{\partial \bar{a}_{2}}{\partial \xi} & -\frac{\partial \bar{a}_{2}}{\partial \eta} \\
\mu \mu_{3}+\mu \mu_{4}+\mu_{3} \mu_{4} & \mu \mu_{3}+\mu \mu_{4}+\mu_{3} \mu_{4} & \mu \mu+\mu \mu_{4}+\mu \mu_{4} & \mu \mu+\mu \mu_{3}+\mu \mu_{3} & \frac{\partial \bar{a}_{3}}{\partial \xi} & \frac{\partial \bar{a}_{3}}{\partial \eta} \\
\mu \mu_{3} \mu_{4} & \mu \mu_{3} \mu_{4} & \mu \mu \mu_{4} & \mu \mu \mu_{3} & -\frac{\partial \bar{a}_{4}}{\partial \xi} & -\frac{\partial \bar{a}_{4}}{\partial \eta}
\end{array}\right)
$$

and the row operations transform it into

$$
\left(\begin{array}{cccccc}
1 & 1 & 1 & 1 & \frac{\partial \bar{a}_{1}}{\partial \xi} & \frac{\partial \bar{a}_{1}}{\partial \eta} \\
\mu_{3}+\mu_{4} & \mu_{3}+\mu_{4} & \mu+\mu_{4} & \mu+\mu_{3} & \star & \star \\
\mu_{3} \mu_{4} & \mu_{3} \mu_{4} & \mu \mu_{4} & \mu \mu_{3} & \star & \star \\
0 & 0 & 0 & 0 & \frac{\partial \bar{P}}{\partial \xi} & \frac{\partial \bar{P}}{\partial \eta}
\end{array}\right)
$$

For the remaining two cases, the same examples as in 3 and essentially the same computations as for $M_{n}^{2}$ show that the codimension of $\widehat{M}^{5} \cap S(z)$ and $\widehat{M}^{8} \cap S(z)$ 
is $\geq 3$ as well. Let us consider for example the case of $\widehat{M}_{n}^{8}$. We choose $\bar{a}=$ $\alpha+\xi \beta+\eta \gamma \in M_{n}^{8}$ with $\alpha=\operatorname{diag}(B, D)$ with

$$
B=\left(\begin{array}{llll}
0 & 1 & 0 & 0 \\
0 & 0 & 0 & 0 \\
0 & 0 & \mu & 1 \\
0 & 0 & 0 & \mu
\end{array}\right),
$$

$\beta, \gamma$ arbitrary matrices in $M_{n}(k)$ and $D=\operatorname{diag}\left(\mu_{5}, \ldots, \mu_{n}\right)$ where all the entries are distinct and different from 0 and $\mu$. We want to find the conditions for $z=$ $\left(x, 0,0, \mu, \mu, \mu_{5}, \ldots, \mu_{n}\right)$ to be smooth. The first $n$ entries of the last row of $J(z)$ vanish and in the last but one row the entries from the $3 \mathrm{~d}$ to the $n$-th also vanish. Consider the $(n-2) \times(n-2)$ submatrix $T$ of $J(z)$ formed by the first $n-2$ rows and the $2,4,5, \ldots, n$th column. By multiplying the first row of $J(z)$ by $\mu$ and substractig it from the second, then multiplying the second by $\mu$ and substracting it from the third, and so on, we transform $T$ into $T^{\prime}=\left(\partial s_{i} / \partial T_{j}\right), 1 \leq i \leq n-2$, $j=2,4,5, \ldots, n$, evaluated at $\left(0, \mu, \mu_{5}, \ldots, \mu_{n}\right)$ where $s_{i}$ is the $i$-th elementary symmetric function in the $n-2$ variables $T_{2}, T_{4}, T_{5}, \ldots, T_{n}$. Since $0, \mu, \mu_{5}, \ldots, \mu_{n}$ are all distinct, $T^{\prime}$ is invertible. This proves that the $2,4, \ldots, n$th columns of $J(z)$ are independent. In the process, the first $n$ entries of the last two rows have become zero. To show that the last two rows are independent from the other ones it suffices now to show that the $2 \times 2$ determinant in the right bottom square does not vanish.

Let us compute the four entries of this determinant. We already saw, in the case of $\widehat{M}_{n}^{2}$ that the last two entries of the last row are $(-1)^{n-1} \frac{\partial \bar{P}}{\partial \xi}(\mu)$ and $(-1)^{n-1} \frac{\partial \bar{P}}{\partial \eta}(\mu)$. The last two entries of the last but one row are, up to sign,

$\frac{\partial \bar{a}_{n-1}}{\partial \xi}+\frac{\partial \bar{a}_{n-2}}{\partial \xi} \mu+\cdots+\frac{\partial \bar{a}_{1}}{\partial \xi} \mu^{n-1}$ and $\frac{\partial \bar{a}_{n-1}}{\partial \eta}+\frac{\partial \bar{a}_{n-2}}{\partial \eta} \mu+\cdots+\frac{\partial \bar{a}_{1}}{\partial \eta} \mu^{n-1}$

which can be computed as

$$
\frac{\frac{\partial \bar{P}}{\partial \xi}(\mu)-\frac{\partial \bar{a}_{n}}{\partial \xi}}{\mu} \text { and } \frac{\frac{\partial \bar{P}}{\partial \eta}(\mu)-\frac{\partial \bar{a}_{n}}{\partial \eta}}{\mu}
$$

Hence, up to a nonzero factor, the determinant we want is

$$
\operatorname{det}\left(\begin{array}{cc}
\frac{\frac{\partial \bar{P}}{\partial \xi}(\mu)-\frac{\partial \bar{a}_{n}}{\partial \xi}}{\mu} & \frac{\frac{\partial \bar{P}}{\partial \eta}(\mu)-\frac{\partial \bar{a}_{n}}{\partial \eta}}{\mu} \\
\frac{\partial \bar{P}}{\partial \xi}(\mu) & \frac{\partial \bar{P}}{\partial \eta}(\mu)
\end{array}\right)=-\frac{1}{\mu} \operatorname{det}\left(\begin{array}{cc}
\frac{\partial \bar{a}_{n}}{\partial \xi} & \frac{\partial \bar{a}_{n}}{\partial \eta} \\
\frac{\partial \bar{P}}{\partial \xi}(\mu) & \frac{\partial \bar{P}}{\partial \eta}(\mu)
\end{array}\right)
$$

We can now compute $\bar{P}$. Using Lemma 5.3 and writing $\bar{a} \in M_{n}(\bar{R})$ as

$$
\operatorname{diag}\left(J_{8}, \mu_{5}, \ldots, \mu_{n}\right)+\left(\bar{a}_{i, j}\right)
$$

we find that $\bar{P}(T)$ is

$$
\begin{aligned}
& \left(T^{2}-\left(\bar{a}_{1,1}+\bar{a}_{2,2}\right) T-\bar{a}_{2,1}\right)\left(T^{2}-\left(2 \mu+\bar{a}_{3,3}+\bar{a}_{4,4}\right) T+\mu^{2}\right. \\
& \left.\quad+\mu\left(\bar{a}_{3,3}+\bar{a}_{4,4}\right)-\bar{a}_{4,3}\right) P_{D}(T)
\end{aligned}
$$


where $P_{D}$ is the characteristic polynomial of $\operatorname{diag}\left(\mu_{5}, \ldots, \mu_{n}\right)$. Denoting by $c$ the constant term of $P_{D}(T)$, we can compute the entries of the determinant above. Since

$$
\bar{a}_{n}=\left(-\bar{a}_{2,1}\right)\left(\mu^{2}+\mu\left(\bar{a}_{3,3}+\bar{a}_{4,4}\right)-\bar{a}_{4,3}\right) c=-\bar{a}_{2,1} \mu^{2} c
$$

and

$$
\bar{P}(\mu)=\left(\mu^{2}-\left(\bar{a}_{1,1}+\bar{a}_{2,2}\right) \mu-\bar{a}_{2,1}\right)\left(-a_{4,3}\right) \bar{P}(\mu)=-\mu^{2} \bar{a}_{4,3} \bar{P}(\mu)
$$

the determinant in $(\dagger)$ is, up to a constant nonzero factor,

$$
\left(\begin{array}{ll}
\beta_{2,1} & \gamma_{2,1} \\
\beta_{4,3} & \gamma_{4,3}
\end{array}\right)
$$

and in the example given this determinant is $\neq 0$.

The rest of the proof of Theorem 8.1 is exactly the same as in Sect. 3 .

Acknowledgments. The authors acknowledge support from NSF DMS-0653382 and from Max-Planck Institut für Mathematik, Bonn. We thank Jean-Louis Colliot-Thélène, Aise Johan de Jong, and David Saltman for several discussions.

Open Access This article is distributed under the terms of the Creative Commons Attribution Noncommercial License which permits any noncommercial use, distribution, and reproduction in any medium, provided the original author(s) and source are credited.

\section{References}

[1] Bourbaki, N.: Algèbre, Chapitre IV, Polynômes et fractions rationnelles. Masson, Paris (1981)

[2] Colliot-Thélène, J.-L.: Algèbres simples centrales sur les corps de fonctions de deux variables (d'après A. J. de Jong), Séminaire Bourbaki, juin 2005, Exp. No. 949. Astérisque 307, 379-413 (2006)

[3] Ekedahl, T., Laksov, D.: Splitting algebras, symmetric functions and Galois theory. J. Algebra Appl. 4, 59-75 (2004)

[4] Fried, M.D., Jarden, M.: Field Arithmetic, Ergebnisse der Mathematik und ihrer Grenzgebiete. Springer, New York (2005)

[5] Grothendieck, A.: Éléments de géométrie algébrique (rédigés avec la collaboration de Jean Dieudonné) : IV. Étude locale des schémas et des morphismes de schémas, Troisième partie. Publications Mathématiques de l'IHÉS 28, 5-255 (1966)

[6] Hartshorne, R.: Algebraic Geometry, Graduate Texts in Mathematics, vol. 52. SpringerVerlag, New York (1977)

[7] Jantzen, J.C.: Nilpotent orbits in representation theory. In: Ørsted, B., Anker, J.-Ph. (eds.) Lie Theory: Lie Algebras and Representations, pp. 1-211. Birkhäuser, Boston (2004)

[8] Van den Bergh, M.: Notes on de Jong's period=index theorem for central simple algebras over fields of transcendence degree two. Preprint (2007). http://archiv.org/abs/0807. 1403 